\title{
3 Vertrauen in Journalismus
}

Unter Online-Bedingungen hat sich also die Rolle des Journalismus gewandelt. Journalistische Medien sind nicht mehr die einzigen Gatekeeper, die darüber entscheiden, welche Informationen an die Öffentlichkeit gelangen und welche nicht. Neben journalistische Medien treten neue Informationsquellen (Alternativmedien, öffentliche Bürgerkommunikation, Originalquellen), die Informationen über aktuelle politische und gesellschaftliche Ereignisse bieten und damit in Konkurrenz und/oder Komplementarität zum Journalismus treten. Diese Entwicklung ist potenziell folgenreich für das Vertrauen in journalistische Medien. Im folgenden Kapitel werden daher zunächst die Grundlagen für das Verständnis von Vertrauen in Journalismus gelegt. Dazu wird die Forschung zu Vertrauen in (journalistische) Medien von den verwandten Bereichen der Glaubwürdigkeitsforschung und der Forschung zur Wahrnehmung journalistischer Qualität abgegrenzt. Im Anschluss wird der Vertrauensbegriff theoretisch geklärt und auf das Vertrauen in journalistische Medien angewendet. Vertrauen in Journalismus wird dabei verstanden als die Bereitschaft, Risiken gegenüber journalistischen Medien einzugehen (Hanitzsch, van Dalen, \& Steindl, 2017, S. 3). Es äußert sich darin, dass Rezipient innen die Informationen journalistischer Medien in ihr Meinungs-, Einstellungs- und Verhaltensspektrum übernehmen. Anschließend werden konzeptuell-methodische Ansätze der Forschung zu Medienvertrauen systematisiert und Forschungsdefizite in diesem Bereich aufgearbeitet. Das Kapitel schließt mit einer Betrachtung der Entwicklung des Vertrauens in Journalismus in Deutschland.

\subsection{Forschungsbereiche zu Medienbewertungen}

Die Kommunikationswissenschaft hat sich intensiv und aus verschiedenen Perspektiven mit Medienbewertungen durch das Publikum befasst (Schweiger, 2007, S. 247). Die Forschung mit explizitem Bezug zu Vertrauen in journalistische Medien ist nur ein Teil dieses Forschungsfeldes, weist jedoch vielfältige Überschneidungen mit anderen Bereichen auf. Es lassen sich im Wesentlichen drei grobe Forschungsbereiche identifizieren, die sich in ihren theoretischen Konzepten und typischen Forschungsfragen unterscheiden. 
Die Forschung zu Medienvertrauen steht in der vorliegenden Studie im Mittelpunkt. Darunter werden sämtliche Ansätze gefasst, die Bewertungen von Medien und Einstellungen gegenüber Medien unter dem Begriff des Vertrauens behandeln und sich dabei - mal mehr, mal weniger intensiv - theoretisch auf sozialwissenschaftliche Vertrauenskonzepte beziehen. Dabei wird für die Zwecke dieser Arbeit Medienvertrauen als Sammelbegriff für jegliches Vertrauen in Medien oder Mediengattungen verstanden, unabhängig davon, ob es sich um journalistische Medien handelt (auch wenn das implizit meist der Fall ist). Dieser Forschungsbereich ist von einer Vielzahl von Begriffen und unterschiedlich definierten Konzepten gekennzeichnet, die nicht trennscharf sind. So untersuchen internationale Studien etwa ,media skepticism“ (Tsfati \& Cappella, 2003), ,confidence in news media' (Johnson \& Kaye, 2014), ,media cynicism' (Carr, Barnidge, Lee, \& Tsang, 2014) oder ,media distrust ${ }^{\star}$ (Ladd, 2010). Im deutschsprachigen Raum sind ,Vertrauen in Journalismus“ (Kohring, 2004) oder ,Medienvertrauen' (Reinemann et al., 2017) verbreitet.

Eng damit verbunden ist die Forschung zur Wahrnehmung journalistischer Qualität. Dieser Bereich beschäftigt sich damit, wie Rezipient_innen bestimmen, was aus ihrer Sicht qualitativ hochwertiger Journalismus ist und wie sie seine Qualität bewerten. Die Forschung zu Qualitätswahrnehmungen weist vor allem deshalb sehr starke Überschneidungen mit der Vertrauensforschung auf, weil Vertrauen in Medien häufig über Qualitätswahrnehmungen operationalisiert wird, Qualitätswahrnehmungen aber auch als Einflussfaktoren auf Vertrauen konzipiert werden (dazu mehr in Kap. 3.4.2). Da Qualitätswahrnehmungen in Kap. 4.2.3 ausführlich behandelt werden, wird hier zunächst nicht näher darauf eingegangen. In den Bereich der Qualitätswahrnehmungen kann auch die Forschung zum Hostile-Media-Effekt eingeordnet werden. Sie beschäftigt sich mit dem empirisch gut belegten Befund, dass Menschen die Berichterstattung zu einem kontroversen Thema als negativ gegenüber ihrer eigenen Meinung zum Thema wahrnehmen (Perloff, 2015; Vallone, Ross, \& Lepper, 1985).

Die Glaubwürdigkeitsforschung ist in der Kommunikationswissenschaft eines der ältesten und prominentesten Forschungsfelder (Metzger, Flanagin, Eyal, Lemus, \& Mccann, 2003, S. 294). Viele Ansätze der Forschung zu Vertrauen in Medien haben daher ihren Ursprung in diesem Forschungszweig, was ihn zu einem zentralen Ausgangspunkt für die Auseinandersetzung mit Vertrauen in Journalismus macht (Kohring \& Matthes, 2007, S. 232). Daher geht das folgende Kapitel ausführlicher auf die Glaubwürdigkeitsforschung ein. Tabelle 3 fasst die angesprochenen Ansätze zusammen.

In dieser Arbeit wird das Verständnis vertreten, dass Vertrauen in Journalismus als ein langfristiges, übergeordnetes Konstrukt fungiert, das beschreibt, inwiefern Rezipient_innen bereit sind, die Informationen journalistischer Medien 
Tabelle 3: Forschungsbereiche zu Medienbewertungen

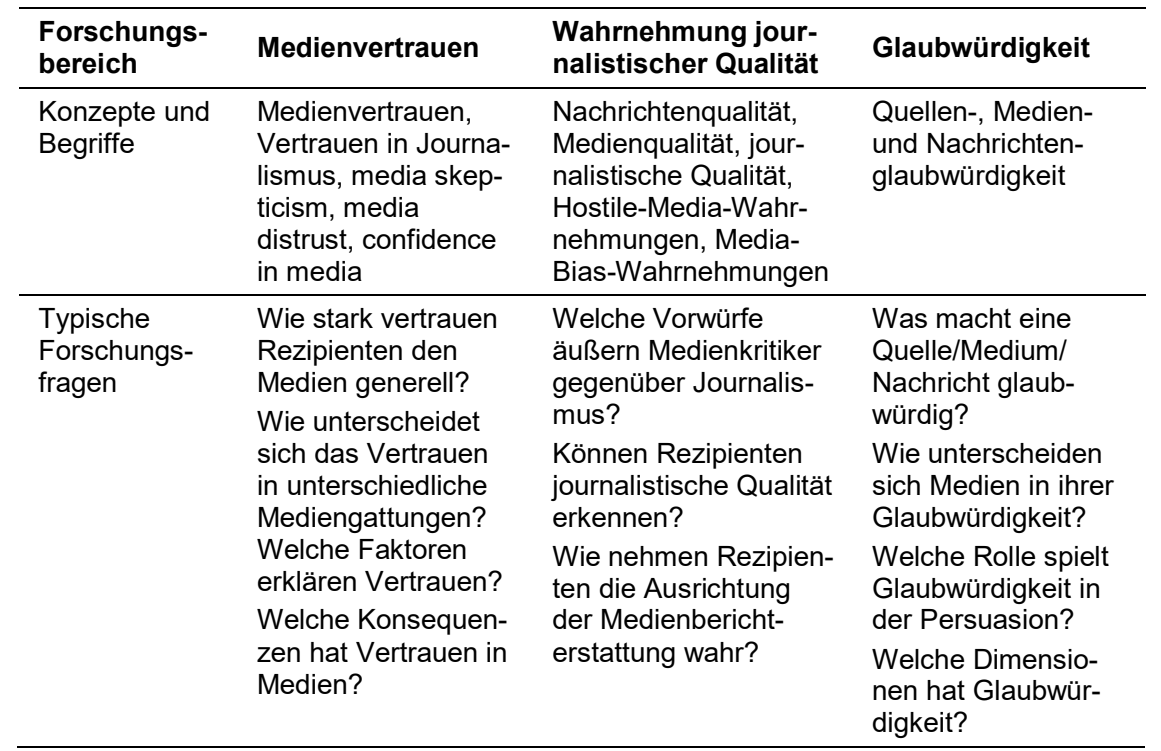

in ihr Meinungs-, Einstellungs- und Verhaltensspektrum zu übernehmen. Vertrauen ist damit auf die Zukunft gerichtet. Qualitätswahrnehmungen sind hingegen dem Vertrauen vorgelagert und beziehen sich auf die Vergangenheit, weil sie auf Basis vergangener Erfahrungen mit Medien getroffen werden. Glaubwürdigkeit wird als eine spezifische Qualitätswahrnehmung verstanden (wahrgenommene Richtigkeit) und damit ebenso wie Qualitätswahrnehmungen als dem Vertrauen vorgelagertes Konstrukt aufgefasst. Die folgenden Kapitel klären dieses Verständnis detaillierter und gehen auf das Verhältnis der drei Ansätze ein.

\subsection{Glaubwürdigkeit}

Glaubwürdigkeit kann mit Metzger und Flanagin (2017, S. 446) als „,believability of information" definiert werden. Glaubwürdigkeit ist damit eine Zuschreibung durch die Rezipient_innen, denn keine Quelle ist an sich glaubwürdig (Schweiger, 2007, S. 247). Gemeinhin werden in der Literatur drei Formen von Glaubwürdigkeit unterschieden (Metzger et al., 2003, S. 296; Appelman \& Sundar, 2016, S. 60): 
1) Quellenglaubwürdigkeit (source credibility),

2) Medienglaubwürdigkeit (media credibility),

3) die Glaubwürdigkeit einzelner Nachrichtenartikel (message credibility).

Zur Quellenglaubwürdigkeit: Diese Forschungsrichtung ist der historische Ausgangspunkt der Glaubwürdigkeitsforschung, die mit Hovland und Kollegen und ihren Yale-Studien zur Persuasion begann (Hovland \& Weiss, 1951; Hovland, Janis, \& Kelley, 1953). Sie waren allerdings weniger an Glaubwürdigkeit an sich interessiert, sondern an ihrer Rolle als intervenierende Variable in der Persuasion. Bis heute ist ein zentrales Ergebnis der Yale-Studien einflussreich: Hovland et al. (1953) unterscheiden zwischen expertness (Kompetenz) und trustworthiness (Vertrauenswürdigkeit) einer Quelle, die ihre Glaubwürdigkeit ausmachen. Unter expertness fassen sie ,the extent to which a communicator is perceived to be a source of valid assertions" (Hovland et al., 1953, S. 21), trustworthiness meint hingegen ,the degree of confidence in the communicator's intent to communicate the assertions he considers most valid" (Hovland et al., 1953, S. 21). Die Wahrnehmung von Kompetenz bei einer Quelle bedeutet also, dass sie in der Lage ist, korrekte Informationen widerzugeben (,capable of transmitting valid statements“, Hovland et al., 1953, S. 21). Wird eine Quelle nicht als kompetent wahrgenommen, werden falsche Informationen durch die Quelle also als unabsichtliche Fehlinformation gewertet. Bei hoher Vertrauenswürdigkeit wird davon ausgegangen, dass die Quelle die korrekten Informationen auch mitteilen will (Schweiger, 2007, S. 260). Bei falschen Informationen wird also eine Manipulationsabsicht und damit absichtliche Desinformation unterstellt (,motivated to make nonvalid assertions", Hovland et al., 1953, S. 21). Die Pionierarbeiten der Yale-Gruppe haben damals einen eigenen Forschungszweig begründet, der sich mit der Wahrnehmung und Zuschreibung von Glaubwürdigkeit auf einzelne Kommunikator_innen beschäftigt. Aus der Forschung zur Quellenglaubwürdigkeit sind zwei weitere Forschungsstränge hervorgegangen: Medien- und Nachrichtenglaubwürdigkeit.

Besonders relevant für das Erkenntnisinteresse dieser Arbeit ist die Forschung zur Medienglaubwürdigkeit. Dabei geht es um die Glaubwürdigkeit unterschiedlicher Mediengattungen im Vergleich (Schweiger, 2007, S. 259). Die prominenteste Abfrage ist die sogenannte Roper-Frage, die seit 1959 für das Television Information Office in den USA in Studien von Roper (1978) verwendet wurde und auch weltweit Einsatz findet. Befragte sollen dabei angeben, welcher Mediengattung sie eher glauben, wenn sie unterschiedliche Versionen einer Nachricht erhalten. Diese Abfrage kam von 1970 bis 1995 auch in Deutschland in der ARD/ZDF-Langzeitstudie Massenkommunikation zum Einsatz (Berg \& Kiefer, 1996, S. 252). In Deutschland wie in anderen Ländern lag hier meistens das Fernsehen vorne, 1970 entschieden sich 75 Prozent der Befragten dafür, nur 
12 Prozent für die Tageszeitung (Berg \& Kiefer, 1996, S. 252). Der Vorsprung des Fernsehens wurde lange darauf zurückgeführt, dass es durch den Einsatz von Filmaufnahmen mehr Authentizität aufweist (Kohring, 2001, S. 16). Allerdings wurde in der Kritik an der Roper-Frage auch darauf hingewiesen, dass unterschiedliche Mediengattungen nur eingeschränkt vergleichbar sind: Menschen basieren etwa ihre Bewertung des Fernsehens stärker auf einzelnen Journalist_innen oder Nachrichtensprecher_innen, wohingegen die Tageszeitung eher als Institution wahrgenommen wird, was Glaubwürdigkeitsunterschiede erklären kann (Newhagen \& Nass, 1989, S. 278). In den Erhebungen der Langzeitstudie Massenkommunikation nach 1970 zeigt sich dennoch ein Imagewandel der Mediengattungen: Die Tageszeitung konnte in der Glaubwürdigkeit stetig zulegen und war 1995 bereits für 31 Prozent der Deutschen das glaubwürdigste Medium (Berg \& Kiefer, 1996, S. 252). Hierin spiegeln sich wohl vor allem Veränderungen im Mediensystem durch die Einführung des dualen Rundfunks. Seit dem Jahr 2000 wird die Glaubwürdigkeit der Mediengattungen in der Langzeitstudie Massenkommunikation mit einer Ranking-Abfrage als Teil mehrerer Image-Dimensionen erhoben (Krupp \& Breunig, 2016, S. 148). Hier liegt die Tageszeitung vorne, die im Jahr 2015 für 35 Prozent der Befragten das glaubwürdigste Medium war, das Fernsehen ist für 31 Prozent am glaubwürdigsten. Dabei ist bei beiden ein leichter Abwärtstrend zu beobachten: Im Jahr 2000 befanden noch 36 Prozent das Fernsehen als glaubwürdigstes Medium, 40 Prozent die Tageszeitung, das Internet hat von 8 Prozent auf 15 Prozent zugelegt (Breunig \& van Eimeren, 2015, S. 523).

Die Roper-Frage und verwandte Abfragemethoden von Medienglaubwürdigkeit wurden und werden allerdings stark kritisiert (Kohring, 2001, S. 18). Die Abfrage von technischen Medienkanälen greift zu kurz, denn Menschen denken bei ,Fernsehen' und ,Tageszeitung' natürlich an sehr unterschiedliche Dinge. Werden die Medien stärker segmentiert, zeigt sich vor allem ein Glaubwürdigkeitsvorsprung des öffentlich-rechtlichen Rundfunks und von Tages- und Wochenzeitungen, wohingegen privates Fernsehen und Boulevardzeitungen negativer gesehen werden (Bayerischer Rundfunk, 2016, S. 26; Krupp \& Breunig, 2016, S. 155). Bei Fernsehen und Tageszeitung kann noch argumentiert werden, dass Rezipient_innen vermutlich an ein relativ überschaubares, einheitliches ,relevant set' prominenter Medienmarken denken, wenn sie nach Mediengattungen gefragt werden. Spätestens bei der Glaubwürdigkeit ,des Internets` ist diese Abfrage aber relativ gehaltlos, denn das Internet vereint so gut wie alle Medienmarken und -gattungen (Metzger et al., 2003, S. 311).

Die Forschung zur Nachrichtenglaubwürdigkeit verschiebt das Bezugsobjekt weg von der Quelle der Information hin zur Information selbst: Sie fragt also danach, für wie glaubwürdig Rezipient innen einen einzelnen Text oder Beitrag halten. Diese Forschungsrichtung überschneidet sich sehr stark mit der 
vor allem im deutschen Sprachraum verbreiteten Forschung zur Wahrnehmung journalistischer Qualität durch das Publikum (Voigt, 2016; Urban \& Schweiger, 2014; Jungnickel, 2011).

In allen drei Bereichen der Glaubwürdigkeitsforschung bilden faktorenanalytische Ansätze einen Forschungsschwerpunkt (Appelman \& Sundar, 2016, S. 59). Sie haben zum Ziel, das Konstrukt Glaubwürdigkeit auf seine zugrundeliegenden Dimensionen zu reduzieren (Schweiger, 2007, S. 259; Kohring \& Matthes, 2007, S. 235; Prochazka \& Schweiger, 2018, S. 4). Einig ist sich diese Forschungsrichtung nur in der Feststellung, dass Glaubwürdigkeit ein mehrdimensionales Konstrukt ist. Es gibt jedoch keinen Konsens dazu, welche Dimensionen das genau sind. In allen drei Bereichen der Glaubwürdigkeitsforschung gibt es unterschiedliche Skalen mit verschiedenen Items und einer unterschiedlichen Anzahl an Faktoren, so dass Glaubwürdigkeit je nach Studie eine nahezu beliebige Anzahl an Dimensionen umfassen kann (Yale, Jensen, Carcioppolo, Sun, \& Liu, 2015, S. 154; Appelman \& Sundar, 2016, S. 60). Das hat der Glaubwürdigkeitsforschung die Kritik eingebracht, dass es vor allem an einer tragfähigen theoretischen Fundierung fehlt, die eine Ableitung von Dimensionen und ihre Operationalisierung erlaubt (Metzger et al., 2003, S. 309; Hellmüller \& Trilling, 2012, S. 2). Unterschiedliche Faktorenstrukturen sind daher vor allem der willkürlichen Auswahl von Items geschuldet.

Zusätzlich ist die Glaubwürdigkeitsforschung nicht klar von der Forschung $\mathrm{zu}$ Vertrauen abgegrenzt. Glaubwürdigkeit und Vertrauen werden häufig synonym verwendet (z. B. Bentele, 1994; Kiousis, 2001; Lee, 2010), Vertrauen wird als Dimension von Glaubwürdigkeit verstanden (Metzger \& Flanagin, 2017, S. 446) oder Glaubwürdigkeit als Dimension von Vertrauen (Bentele \& Seidenglanz, 2015, S. 412; Kohring, 2004, S. 174). Auch wenn einige Autor_innen die theoretischen Unterschiede zwischen den beiden Konstrukten betonen oder das jeweils andere begrifflich ignorieren, sind sie spätestens empirisch nicht mehr trennscharf: Skalen aus der Glaubwürdigkeitsforschung, die wiederum auf Qualitätswahrnehmungen basieren, werden zur Operationalisierung von Vertrauen herangezogen (Prochazka \& Schweiger, 2018, S. 3). Diese mangelnde theoretische wie empirische Trennung von Vertrauen und Glaubwürdigkeit kommt nicht von ungefähr. Vertrauen und Glaubwürdigkeit sind trotz ihrer Unterschiede theoretisch eng miteinander verknüpft und korrelieren selbstverständlich auch empirisch - wer Medien für glaubwürdig hält, wird ihnen eher vertrauen und umgekehrt. In dieser Arbeit werden die beiden Begriffe jedoch theoretisch und konzeptuell voneinander abgegrenzt. Glaubwürdigkeit meint den Grad, mit dem Informationen eines Bezugsobjekts als wahr erachtet werden und wird daher auch als wahrgenommene Richtigkeit bezeichnet (Kohring, 2001, S. 18). Glaubwürdigkeit ist damit dem Vertrauen vorgelagert (Kohring, 2004, S. 17; Jackob, 2009, S. 385). Das Konzept Vertrauen ist entgegen der häufig synonymen Ver- 
wendung also nicht gleichbedeutend mit Glaubwürdigkeit, sondern geht konzeptuell weit darüber hinaus. Das folgende Kapitel klärt den Vertrauensbegriff theoretisch und wendet ihn auf die Vertrauensbeziehung zwischen journalistischen Medien und ihrem Publikum an.

\subsection{Vertrauen}

\subsubsection{Grundlagen des Vertrauensbegriffs}

In der Literatur besteht Einigkeit darüber, dass Vertrauen als sozialer und psychologischer Mechanismus für Individuen und die Gesellschaft von herausragender Bedeutung ist. Vertrauen ist nach Luhmann (2014) eine der unabdingbaren Voraussetzungen, die soziales Handeln überhaupt erst möglich machen. Er hält fest, der Mensch könne ,[o]hne jegliches Vertrauen [...] morgens sein Bett nicht verlassen“ (S. 1). Bereits Simmel ${ }^{7}$ (1908) bezeichnete das Vertrauen in einer der frühesten soziologischen Auseinandersetzungen mit dem Konstrukt als „eine der wichtigsten synthetischen Kräfte innerhalb der Gesellschaft" (S. 346).

Vertrauen wird in der Regel als Relation zwischen zwei Akteuren begriffen: Sie besteht zwischen einem Vertrauensobjekt (auch: Vertrauensnehmer), also wem oder was vertraut wird, und einem Vertrauenssubjekt (auch: Vertrauensgeber), also wer vertraut (Gambetta, 1998, S. 217). Luhmann (2014, S. 27) begründet die besondere Bedeutung von Vertrauen damit, dass es als sozialer Mechanismus zur Reduktion von Komplexität in der Gesellschaft fungiert. Vertrauen dient in erster Linie dazu, „Nichtwissen [zu] kompensieren“ (Kohring, 2004, S. 83). Diese Idee geht zurück auf Simmel (1908), der Vertrauen als „eine[n] mittleren Zustand zwischen Wissen und Nichtwissen [...]“ (S. 346) bezeichnet: „Der völlig Wissende braucht nicht zu vertrauen, der völlig Nichtwissende kann vernünftigerweise nicht einmal vertrauen." (S. 346). Vertrauen reduziert also soziale Komplexität, indem es bestimmte Handlungsoptionen nahelegt und andere ausschließt. Es ermöglicht damit, die Kluft zwischen Unsicherheit und Handlung zu überbrücken (Luhmann, 2014, S. 30). Vertrauen sorgt damit für (zumindest wahrgenommene) Handlungssicherheit, denn es generalisiert Erwartungen an zukünftige Handlungen (Hanitzsch et al., 2017, S. 3). In

7 Simmels Ausführungen zu Vertrauen beschränken sich auf einige wenige Seiten in ,Philosophie des Geldes‘ (2009) [1900]) und ,Soziologie‘ (1908), sind aber noch heute mit Gewinn zu lesen und haben so gut wie alle folgenden Vertrauenstheoretiker beeinflusst (Möllering, 2001, S. 408). So gut wie alle zentralen Annahmen der aktuellen Vertrauensforschung lassen sich bereits in Simmels Arbeiten finden. 
einer kontingenten ${ }^{8}$ Welt mit unzähligen Handlungsoptionen bietet Vertrauen damit Orientierung und Hilfe bei Entscheidungen (Quandt, 2012, S. 8). Ohne ein Mindestmaß an Vertrauen wären diese entweder nur mit enormem Aufwand zu treffen (indem alle Unsicherheit ausgeschlossen wird) oder würden bei völliger Unwissenheit zu einer reinen Zufalls- oder rationalen Wahrscheinlichkeitsentscheidung. Das bedeutet auch, dass Vertrauen niemals absolut werden, d. h. völlige Sicherheit garantieren kann und niemals völlig grundlos entgegengebracht wird (Vanacker \& Belmas, 2009, S. 112).

Vertrauen ist allerdings mehr als nur eine ,einfache" Bewertung bzw. ein Urteil über ein Vertrauensobjekt. Vanacker und Belmas (2009) halten fest: „we cannot equate evaluating or judging with trust. Trust is judgment, but a particular kind of judgment, one in which the one who judges, the trustor, has a stake" (S. 112). Es handelt sich also nur um Vertrauen, wenn es für das Vertrauenssubjekt auch ,um etwas geht', wenn also Risiken eingegangen werden:

Vertrauen bezieht sich [...] stets auf eine kritische Alternative, in der der Schaden beim Vertrauensbruch größer sein kann als der Vorteil, der aus dem Vertrauenserweis gezogen wird. Der Vertrauende macht sich mithin an der Möglichkeit übergroßen Schadens die Selektivität des Handelns anderer bewußt und stellt sich ihr. (Luhmann, 2014, S. 28).

Damit ist eine Entscheidung auf der Basis von Vertrauen also immer eine Entscheidung gegen eine mögliche Alternative (Stichwort Kontingenz), die bewusst in Akzeptanz eines Risikos getroffen wird. Dieses Element ist für das Verständnis von Vertrauen zentral, weil es erklärt, warum enttäuschtes Vertrauen nur schwierig wiederhergestellt werden kann: Die bewusste Entscheidung für eine Alternative hat sich dann im Fall der Vertrauensenttäuschung als falsche Entscheidung herausgestellt, die in Zukunft vermieden werden soll. Damit lässt sich Vertrauen auch vom Begriff der Hoffnung abgrenzen. Hoffnung bezieht sich auf Ereignisse, die vom Akteur nicht beeinflusst werden können: „[d]er Hoffende faßt trotz Unsicherheit einfach Zuversicht. Vertrauen reflektiert Kontingenz, Hoffnung eliminiert Kontingenz" (Luhmann, 2014, S. 29).

Insofern ist Vertrauen - auch hier sind sich die Definitionen einig - immer auf die Zukunft gerichtet (Hanitzsch et al., 2017, S. 4). Simmel (1908) beschreibt Vertrauen als ,die Hypothese künftigen [Hervorh. FP] Verhaltens, die sicher genug ist, um praktisches Handeln darauf zu gründen“ (S. 346). Ein Element von

8 Der Begriff der Kontingenz spielt in der Systemtheorie nach Parsons und Luhmann eine wichtige Rolle, er meint die prinzipielle Offenheit bzw. vielfältigen Möglichkeiten sozialer Realität: „Der Begriff bezeichnet mithin Gegebenes (Erfahrenes, Erwartetes, Gedachtes, Phantasiertes) im Hinblick auf mögliches Anderssein; er bezeichnet Gegenstände im Horizont möglicher Abwandlungen. Er setzt die gegebene Welt voraus, bezeichnet also nicht das Mögliche überhaupt, sondern das, was von der Realität aus gesehen anders möglich ist.“(Luhmann, 1987, S. 152) 
Vertrauen ist demnach, dass es basierend auf vergangenen Erfahrungen immer auf Erwartungen gründet, wie sich ein Vertrauensobjekt in Zukunft verhalten wird (statt vieler: Sztompka, 1999, S. 18). Als Hypothese künftigen Verhaltens ist Vertrauen allerdings stark risikobehaftet, denn das zukünftige Verhalten eines Vertrauensobjekts lässt sich nicht sicher voraussagen.

Der zweite Teil von Simmels oben genannter Definition von Vertrauen als Hypothese künftigen Verhaltens bezieht sich darauf, dass auf Vertrauen praktisches Handeln gegründet wird. Diesen Aspekt greift auch Luhmann auf, wenn er als Kern von Vertrauen ausmacht „an fremde Selektionsleistungen eigenes Verhalten anzuschließen“ (Luhmann, 2014, S. 62). Bei einer Vertrauensrelation geht es demnach um die Übertragung von Handlungsverantwortung, die Luhmann (2014) als „riskante Vorleistung“ (S. 27) von Seiten der Vertrauenden beschreibt. Wer vertraut, lässt damit andere für sich entscheiden und geht das Risiko ein, dass diese Entscheidungen negative Konsequenzen für ihn oder sie haben (Kohring, 2004, S. 132). In diesen Elementen liegt auch die wesentliche Abgrenzung zum Begriff der Glaubwürdigkeit: Weil Glaubwürdigkeit prinzipiell die „believability of information“ (Metzger \& Flanagin, 2017, S. 446) bezeichnet, geht es dabei nicht um eine Hypothese künftigen Verhaltens und auch nicht um die Bereitschaft, Handlungen darauf zu gründen. So wird auch deutlich, dass sich Glaubwürdigkeit am besten als eine Voraussetzung von Vertrauen verstehen lässt: Werden die Aussagen einer Person oder Institution als glaubwürdig wahrgenommen, kann ihr Vertrauen entgegengebracht werden, muss aber nicht.

Hanitzsch et al. (2017, S. 3) fassen zusammen, dass Vertrauen 1) zur Reduktion sozialer Komplexität dient, also Handlungsmöglichkeiten eröffnet und andere ausschließt, 2) Risiken enthält, weil das Verhalten eines Vertrauensobjekts nicht vorhergesagt werden kann und 3) auf die Zukunft gerichtet ist und von Erwartungen an ein Vertrauensobjekt abhängt. Diese genannten Eigenschaften von Vertrauen finden sich kondensiert in einer Definition von Mayer, Davis und Schoorman (1995) aus dem Bereich der Organisationspsychologie, die in den vergangenen Jahren vor allem im Bereich der Forschung zu Vertrauen in Medien einige Popularität erlangt hat und zunehmend als grundlegende Vertrauensdefinition in diesem Bereich herangezogen wird (z. B. Hanitzsch et al., 2017, 3; Grosser, Hase, \& Wintterlin, 2017, S. 2; Blöbaum, 2016, S. 6; Engelke, Hase, \& Wintterlin, 2019, S. 3; Fawzi \& Obermaier, 2019, S. 36). Sie soll auch der vorliegenden Untersuchung zu Grunde liegen. Mayer et al. definieren Vertrauen als:

[...] the willingness of a party to be vulnerable to the actions of another party based on the expectation that the other will perform a particular action important to the trustor, irrespective of the ability to monitor or control that other party. (Mayer et al., 1995, S. 712) 
Knapp zusammengefasst ist Vertrauen demnach ,not taking risk per se, but rather a willingness to take risk" (Mayer et al., 1995, S. 712).

Daran anschließend stellt sich die Frage, was Misstrauen ausmacht. Misstrauen erfährt in der Literatur deutlich weniger Aufmerksamkeit als Vertrauen und wird in aller Regel als Gegenteil von Vertrauen behandelt (Tsfati \& Cappella, 2003, S. 506; Engelke, 2018, S. 142). Luhmann (2014) schließt sich dieser Sichtweise an, betont aber, dass Misstrauen nicht nur fehlendes Vertrauen ist. Vielmehr fungiert Misstrauen als „funktionales Äquivalent“ (S. 92) von Vertrauen, denn es dient ebenso der Reduktion von Komplexität. Wer einfach nur nicht vertraut, „stellt die ursprüngliche Komplexität der Geschehensmöglichkeiten wieder her und belastet sich damit." (Luhmann, 2014, S. 93). Misstrauen erfüllt als Gegenpol von Vertrauen also dieselbe Funktion der Reduktion von Komplexität, hat aber andere Konsequenzen für Handlungen. Bei einem Verlust von Vertrauen in einen Vertrauensnehmer verschwindet Vertrauen also nicht einfach, denn es ist als stabilisierender Mechanismus notwendig, um Entscheidungen überhaupt zu ermöglichen. Vielmehr kann Vertrauen einerseits in Misstrauen umschlagen, dass dieselbe Funktion erfüllt, und/oder sich nach einem Vertrauensverlust auf andere Bezugsobjekte richten.

\subsubsection{Vertrauen in journalistische Medien}

Das hier skizzierte Verständnis von Vertrauen lässt sich prinzipiell auf unterschiedliche Vertrauensbeziehungen und Bezugsobjekte anwenden. Bislang wurde Vertrauen relativ unspezifisch als Mechanismus bestimmt, der als Relation zwischen zwei nicht näher bestimmten Akteuren stattfindet. Neser (2016, S. 256) unterscheidet drei Bereiche von Vertrauen: das Selbstvertrauen, das interpersonelle Vertrauen sowie das Institutionenvertrauen. Selbstvertrauen beschreibt den „Glaube[n] oder die Sicherheit, die Fähigkeit zu besitzen, bestimmte Handlungen zu organisieren und auszuführen“ (Jünemann, 2016, S. 192). Interpersonelles Vertrauen beschreibt eine Vertrauensrelation zwischen zwei oder mehr Personen, die sich persönlich kennen. Diese Form des Vertrauens ist in der (sozial)psychologischen Literatur die prominenteste und liegt implizit oder explizit daher auch vielen theoretischen Auseinandersetzungen zu Grunde. Das Konzept des Institutionenvertrauens überträgt den Vertrauensbegriff aus einer Beschreibung interpersonaler Beziehungen auf das Verhältnis zwischen Einzelpersonen und gesellschaftlichen Institutionen, darunter etwa Politik, Wirtschaft, Justiz, Polizei oder auch Medien und Journalismus (Høyer \& Mønness, 2016, S. 2; PytlikZillig et al., 2016, S. 2).

Journalismus kann wie in Kap. 2.1 festgehalten als System auf der Makroebene, als Institution(en) auf der Mesoebene und als journalistische Akteure auf der Mikroebene definiert werden. Aus der Perspektive des Publikums ist zwi- 
schen einem Vertrauen in das System Journalismus und in die Institutionen der journalistischen Medien jedoch kaum zu unterscheiden, denn das System manifestiert sich über seine Institutionen. Vertrauen in das System Journalismus ist also zwar analytisch vom Vertrauen in Medieninstitutionen zu trennen, wird empirisch aber kaum zu unterscheiden sein. Vertrauen in Journalismus wird daher in der Regel als eine Form des Institutionenvertrauens verstanden (Hanitzsch et al., 2017, S. 3; Tsfati \& Cappella, 2003, S. 504; Mishler \& Rose, 2001, S. 40). Gemäß dem oben skizzierten theoretischen Konzept von Vertrauen wird hier Vertrauen in Journalismus als die Bereitschaft von Rezipient_innen verstanden, Risiken gegenüber den Informationen in journalistischen Medien einzugehen (Hanitzsch et al., 2017, S. 3). Vertrauende gehen bei Vertrauen in Journalismus ganz allgemein das Risiko ein, ihre Handlungen auf falschen, unvollständigen oder irrelevanten Informationen beruhen zu lassen (Matthes \& Kohring, 2003, S. 10). Damit ist im Sinne Luhmanns die Möglichkeit großen Schadens eingeschlossen, denn eine Vertrauenshandlung bedeutet dann, dass journalistischen Medien die Handlungsverantwortung in bestimmten Bereichen übertragen wird. Vertrauen in Journalismus zeigt sich also, wenn für Vertrauenssubjekte bestimmte Anschlusshandlungen in Frage kommen, die auf einer Akzeptanz von Informationen aus den Medien beruhen. Das ist besonders bedeutsam, weil die Qualität journalistischer Medien als Vertrauensgüter für die Rezipient_innen kaum überprüfbar ist (Kiefer, 2005, S. 142). Vertrauen in Journalismus ist damit mehr als ,nur' Glaubwürdigkeit, denn es umfasst zusätzlich die Bereitschaft, ein Risiko in Bezug auf ihre Informationen einzugehen. Konkret äußert sich dieses Vertrauen etwa in der Bereitschaft von Personen, die Informationen aus den Medien zur eigenen Meinungsbildung zu einem Thema heranzuziehen, Wahlentscheidungen auf der Grundlage von medienvermittelten Informationen zu treffen, die Informationen in Gesprächen zur Untermauerung der eigenen Position $\mathrm{zu}$ verwenden oder an andere weiterzugeben. Es geht bei Vertrauen in Journalismus also darum, „dem eigenen Handeln journalistische Informationsangebote zu Grunde zu legen." (Matthes \& Kohring, 2003, S. 10) Misstrauen in Journalismus bedeutet dann, sich bei solchen Entscheidungen nicht bzw. nur mit Vorbehalten auf die Medien zu verlassen. Angelehnt an die oben genannten Punkte und an die Glaubwürdigkeitsdefinition von Wirth (1999, S. 55) soll Vertrauen in Journalismus in dieser Studie daher verstanden werden als die Bereitschaft einer Person, journalistische Informationen in das eigene Meinungs-, Einstellungsund Verhaltensspektrum zu übernehmen. 


\subsection{Konzeptuell-methodische Ansätze der Medienvertrauensforschung}

Auch in der Forschung mit explizitem Fokus auf Vertrauen in Medien existieren sehr unterschiedliche konzeptuelle und methodische Herangehensweisen. Das Forschungsfeld ist von einer uneinheitlichen und nicht trennscharfen Verwendung von Begriffen gekennzeichnet, die eine Systematisierung nicht ganz einfach machen. So finden sich etwa sehr ähnliche Definitionen der untersuchten Konstrukte, die allerdings ganz unterschiedlich benannt werden, oder es werden ähnliche Begriffe für definitorisch unterschiedliche Konstrukte gebraucht. Diese Verwirrung setzt sich empirisch fort: Konzeptuell ähnliche Konstrukte werden unterschiedlich operationalisiert oder konzeptuell unterschiedliche Konstrukte sehr ähnlich. Exemplarisch zeigt sich das etwa in den Arbeiten von Tsfati (z. B. 2003a), der das Konzept ,media skepticism‘ untersucht, das er unter anderem als „mistrust toward the mainstream news media“ (S. 160) definiert und zur Messung vornehmlich Items aus einer Glaubwürdigkeitsskala von Gaziano und McGrath (1986) heranzieht, die nichts anderes sind als generalisierte Qualitätswahrnehmungen etablierter journalistischer Medien (sind diese ausgewogen, fair, stellen die Fakten richtig dar, usw.).

Das folgende Kapitel soll in diesem Bereich für etwas mehr Klarheit sorgen, indem konzeptuell-methodische Forschungsansätze im Bereich der Forschung zu Vertrauen in Medien durch Kombinationen aus dem untersuchten Vertrauensobjekt und der Art der Operationalisierung systematisiert werden. Diese Systematik baut auf den Zusammenfassungen des Forschungsbereichs von Schielicke, Mothes und Donsbach (2014, S. 249) sowie Jackob und Schultz et al. (2017, S. 121-122) auf.

Als Vertrauensobjekt werden einerseits spezifische Medien untersucht, also etwa bestimmte Mediengattungen und -marken oder die von den Befragten selbst genutzten Medien (Ansätze 1 und 2). Andererseits wird das Vertrauen in Medien allgemein untersucht, womit in der Regel explizit oder implizit etablierte, journalistische ,Mainstream-Medien“ gemeint sind (Ansätze 3 und 4). In diesem Bereich ist auch die vorliegende Studie verortet.

Das Vertrauen in die jeweiligen Bezugsobjekte (spezifische Medien oder Medien allgemein) wird dann in einer ersten Variante mittels Qualitätswahrnehmungen operationalisiert ${ }^{9}$ (Prochazka \& Schweiger, 2018, S. 3) indem jeweils nach der wahrgenommenen Einhaltung unterschiedlicher journalistischer Qualitätsdimensionen gefragt wird und die Antworten auf diese Fragen dann als

9 Hier werden nur Ansätze von Befragungsstudien systematisiert, die die Medienvertrauensforschung nachvollziehbarer Weise dominieren. 
Tabelle 4: Forschungsansätze in der Medienvertrauensforschung

\begin{tabular}{lll}
\hline & Bezugsobjekt & \\
\hline $\begin{array}{l}\text { Operationalisierung } \\
\text { von Vertrauen }\end{array}$ & Spezifische Medien & $\begin{array}{l}\text { Journalismus/etablierte } \\
\text { Medien }\end{array}$ \\
\hline $\begin{array}{l}\text { Abfrage von Qualitäts- } \\
\text { wahrnehmungen }\end{array}$ & $\begin{array}{l}\text { Ansatz (1): Vertrauen in } \\
\text { spezifische Medien, Abfrage } \\
\text { von Qualitätswahrnehmun- } \\
\text { gen, z. B. Vertrauen in meist- } \\
\text { genutztes Medium (Kohring, }\end{array}$ & $\begin{array}{l}\text { Ansatz (3): Vertrauen in Me- } \\
\text { dien allgemein, Abfrage von } \\
\text { Qualitätswahrnehmungen, } \\
\text { z. B. Studien zu Medienskep- } \\
\text { sis (Tsfati \& Cappella, 2003; }\end{array}$ \\
& $\begin{array}{l}\text { Tsfati \& Peri, 2006) } \\
\text { Ansatz (2): Vertrauen in } \\
\text { spezifische Medien, Global- } \\
\text { abfrage von Vertrauen, z. B. } \\
\text { Vertrauen in TV, Radio, Zei- } \\
\text { tungen, Internet (Eurobaro- } \\
\text { meter, 2014) }\end{array}$ & $\begin{array}{l}\text { Ansatz (4): Vertrauen in Me- } \\
\text { dien allgemein, Globalabfrage } \\
\text { von Vertrauen, z. B. Studien } \\
\text { zu generalisiertem Vertrauen } \\
\text { in Journalismus (Jackob et al., } \\
\text { 2019; Schindler, Fortkord, }\end{array}$ \\
& & $\begin{array}{l}\text { Posthumus, Obermaier, \& } \\
\text { Reinemann, 2018) }\end{array}$ \\
\hline
\end{tabular}

Indikatoren für Vertrauen interpretiert werden (Schielicke et al., 2014, S. 249) (Ansätze 1 und 3). Dieses Vorgehen steht in der Tradition der Glaubwürdigkeitsforschung und den dortigen Versuchen, Medien- oder Quellenglaubwürdigkeit über mehrdimensionale Skalen zu erfassen. In diesem Bereich werden daher auch meistens Items aus Glaubwürdigkeitsskalen übernommen (Prochazka \& Schweiger, 2018, S. 3). Die zweite Variante ist eine Operationalisierung mittels Globaleinschätzung, indem schlicht (meist mit einem Item) direkt nach dem Ausmaß des Vertrauens in bestimmte Bezugsobjekte gefragt wird (Ansätze 2 und 4). Tabelle 4 systematisiert diese unterschiedlichen Ansätze und stellt sie im Überblick dar. Die folgenden Kapitel gehen gesondert auf die beiden zugrundeliegenden Dimensionen ein. Da generalisiertes Vertrauen in journalistische Medien im Mittelpunkt dieser Studie steht, sind vor allem die Forschungsansätze 3 und 4 relevant.

\subsubsection{Spezifisches vs. generalisiertes Vertrauen in Journalismus}

Studien zum Vertrauen in spezifische Medien ziehen unterschiedlich abstrakte Bezugsobjekte heran. Neben dem Vertrauen in einzelne Medien oder Medienmarken wie bei Kohring (2004) wird in der Tradition der Forschung zur Medienglaubwürdigkeit vor allem das Vertrauen in unterschiedliche Mediengattungen verglichen. So untersuchen etwa größere vergleichende Studien wie das Eurobarometer (2014) das Vertrauen in Fernsehen, Radio, Printmedien und In- 
ternet. Die Ergebnisse ähneln strukturell denen zur Medienglaubwürdigkeit (Kap. 3.2) und werden daher hier nicht eingehender referiert.

Auf der anderen Seite stehen Ansätze, die Vertrauen in Medien als ein generalisiertes Vertrauen der Rezipient_innen gegenüber (journalistischen) Medien untersuchen. Diese Studien begreifen das Vertrauensobjekt damit als „abstrakten Kollektivbegriff für etablierte Medien, anstatt auf konkret genutzte oder verfügbare Einzelmedien [...] zu setzen“ (Jackob, Schultz et al., 2017, S. 122). Ein solches generalisiertes Journalismusvertrauen steht im Mittelpunkt dieser Arbeit. Dieses generalisierte Vertrauen in ,die Medien' erscheint zwar auf den ersten Blick plausibel, zunächst sind jedoch zumindest drei grundlegende und miteinander verknüpfte Fragen zu klären:

1) Welche Medien fallen aus theoretischer Perspektive unter den breiten Begriff der etablierten journalistischen Medien, was ist also das Vertrauensobjekt?

2) Existiert ein so verstandenes einheitliches Bild von ,den Medien' tatsächlich auch bei Rezipient_innen und welche Medien umfasst es?

3) Wird diesen Medien ein generalisiertes Vertrauen entgegengebracht oder unterscheidet sich das Vertrauen zu stark je nach Mediengattung oder Marke, so dass die Untersuchung eines generalisierten Medienvertrauens nicht sinnvoll ist?

Zur ersten Frage: Das generalisierte Vertrauen in Journalismus lässt sich am besten als ein Vertrauen gegenüber den Institutionen der Mainstream-Medien beschreiben. Der Begriff ,Mainstream-Medien“ wird häufig in (rechts)alternativen Medien als despektierlicher Kampfbegriff für den etablierten Journalismus verwendet, was seine Verwendung durch die negativen Konnotationen nicht ganz unproblematisch macht. Er ist dennoch geeignet, den hier interessanten Bereich des Journalismus genauer zu bestimmen und scheint in jüngster Zeit auch akademisch populär zu werden. Jackob, Quiring und Schemer (2017) bezeichnen etwa den „,medialen Mainstream“ als „,[eine] abstrakt[e] mentale Repräsentation" (S. 229) und fassen darunter

[...] jene Form von gesellschaftlich prävalenten Nachrichtenmedien, die am Weitesten verbreitet, am Leichtesten verfügbar und strukturell wie inhaltlich am Ähnlichsten gestaltet sind - und die von der Mehrheit der Bürger als Medien erinnert, benannt und genutzt werden, wenn von Medien die Rede ist. (Jackob, Quiring et al., 2017, S. 229)

Schweiger (2017) beschreibt die Entwicklung von „Mainstream-Medien“ (S. 39, Hervorh. i.O.) als direkte Konsequenz der Abhängigkeit von Werbeerlösen und Reichweiten, da sich Medien im Streben nach einem möglichst großen Publikum am „Mehrheitsgeschmack der Gesellschaft, eben am Mainstream“ (S. 39) orientieren müssen. Die Berichterstattung orientiert sich dann inhaltlich an der politi- 
schen, sozialen und ökonomischen Mitte der Gesellschaft und blendet andere Positionen tendenziell aus, auch wenn Medienhäuser nach wie vor politische Färbungen aufweisen. Krüger $(2016,2017)$ hat den Mainstream-Begriff ebenfalls aufgegriffen, spricht aber nicht pauschal von Mainstream-Medien, weil der Begriff nahelegt, dass diese Medien immer konsonant berichten (Krüger, 2017, S. 251). Auch in Mainstream-Medien tauchen allerdings immer wieder andere Themen und Ereignisdarstellungen auf, die jedoch nicht denselben Beachtungsgrad erfahren wie die orthodoxe ,Mainstream-Meinung'. Er verwendet daher den Begriff des „Medien-Mainstreams“ und meint damit

[...] das Phänomen, dass es eine Anzahl von Themen und Meinungen gibt, die in einem bestimmen Zeitraum in den Leitmedien dominieren und damit eine Hauptströmung oder eine Hauptrichtung bilden, wobei mal die eine Zeitung, mal die andere Sendung vom Themen- oder Meinungs-Mainstream abweichen kann. (Krüger, 2017, S. 251)

Unter diese Vorstellung vom medialen Mainstream fallen also die großen, bekannten und reichweitenstarken Medienmarken wie die öffentlich-rechtlichen und privaten Rundfunksender, noch immer Tageszeitungen (trotz sinkender Auflagen), Wochenzeitungen sowie die jeweiligen Online-Portale dieser Anbieter. Diese machen selbstverständlich immer noch einen nicht klar abzugrenzenden Bereich innerhalb der Vielzahl an (journalistischen) Medien aus, so dass vor allem die Perspektive der Rezipient innen in den Vordergrund rückt.

Das führt zur zweiten Frage, nämlich inwiefern auch in den Köpfen der Rezipient innen ein so einheitliches Bild vom medialen Mainstream existiert und welche Medien es umfasst. Es gibt eine Reihe empirischer Hinweise darauf, dass Rezipient_innen tatsächlich ein generalisiertes Bild von ,den Medien' im Kopf haben. In den USA hat Ladd (2012, S. 96-103) in offenen Fragen erhoben, woran Menschen denken, wenn sie Fragen zu ihrem Medienvertrauen beantworten. In der Auswertung zu den genannten Medienmarken oder -gattungen zeigt sich, dass 80 Prozent der Befragten kein spezifisches Medium nennen, sondern allgemein von den Medien sprechen. Wenn alternative Medienanbieter genannt wurden, nehmen die Befragten sie offenbar im starken Kontrast zu etablierten journalistischen Medien wahr:

Overall, very few people think about any specific media outlets when asked these media evaluations questions [...]. Those who think about alternative media outlets consider them as a contrast to the institutional news outlets and professional journalism in general. They base their evaluations of 'the press,' 'the news media,' or just 'the media' on their reactions to these latter objects. (Ladd, 2012, S. 103)

Zum gleichen Schluss kommen Tsfati und Cappella (2005), die basierend auf Gruppendiskussionen für die Existenz einer generalisierten Vorstellung etablierter Medien argumentieren: ,people have some mental schema for what "the 
news media' are, and [...] this construct operates whenever people confront this construct in survey (or any other) contexts." (S. 268). In einer Studie in Schweden (einem mit Deutschland vergleichbaren Mediensystem) kommt Kaun (2014) basierend auf einer Tagebuch-Erhebung und qualitativen Leitfadeninterviews zu dem Ergebnis, dass Rezipient_innen ,die Medien“als generalisierte Bezugsobjekte in ihren Schilderungen verwenden und ein relativ einheitliches Verständnis davon haben, welche Medien dazuzählen: Sie verstehen darunter vor allem große, etablierte Medienhäuser wie den öffentlich-rechtlichen Rundfunk oder große Tageszeitungen. Auch Inhaltsanalysen zu Nutzerwahrnehmungen von Journalismus in Online-Leserkommentaren zeigen, dass Rezipient innen ihre Kritik an Journalismus generalisiert und pauschal äußern. Sie verleihen darin offenbar einer Einstellung gegenüber ,den Medien “ als solchen Ausdruck, was sich vor allem darin zeigt, dass sie unklare und pauschale Bezugsobjekte verwenden (Prochazka \& Schweiger, 2016, S. 463).

Zur dritten Frage: Gibt es zu diesem einheitlichen Bild von etablierten Medien auch ein generalisiertes Vertrauen? Studien zum Vertrauen in Fernsehen, Radio, Zeitung und Online-Quellen zeigen je nach Gattung durchaus unterschiedliche Werte, auch die Forschung zur Medienglaubwürdigkeit und Medienimages zeigen Differenzen. Generell zeigt sich außerdem, dass Abfragen allgemeiner Kategorien wie ,die Medien“ oder , die Presse' relativ geringe Vertrauenswerte zeigen, während konkreten Sendungen, Zeitungen oder Magazinen stärker vertraut wird (Blöbaum, 2018, S. 606; Köcher, 2016, S. 8). Rezipient_innen könnten also zwar ein relativ einheitliches Bild vor Augen haben, wenn von ,den Medien' die Rede ist, aber dennoch differenzierte Vertrauenseinschätzungen zu einzelnen Elementen dieser Globalkategorie haben. Als eine der wenigen Studien untersuchte Kiousis (2001), wie die Einschätzungen zur Glaubwürdigkeit (in der Studie synonym mit Vertrauen verwendet) einzelner Mediengattungen zusammenhängen. Er kommt zu dem Schluss, dass Glaubwürdigkeitseinschätzungen hoch miteinander korrelieren:

Survey participants seemed to perceive all of the media channels in the same direction, indicating that people probably have an overall perception of news credibility that only slightly fluctuates across media. [...] Therefore, perceptions of news credibility are fairly uniform, although some distinctions are made among media channels. (Kiousis, 2001, S. 396)

Die Vertrauenslevels unterschiedlicher Mediengattungen mögen sich also im Durchschnitt und auf Individualebene unterscheiden, hängen aber stark zusammen. Wer Fernsehnachrichten nicht vertraut, vertraut auch Zeitungsnachrichten weniger, obwohl das Vertrauen in die eine oder andere Mediengattung absolut höher sein kann. Das weist darauf hin, dass Rezipient innen eine generalisierte Einstellung zu journalistischen Medien ,im Großen und Ganzen` besitzen. Es 
erscheint daher sinnvoll, ein solches generalisiertes Vertrauen genauer zu untersuchen.

\subsubsection{Operationalisierung: wahrgenommene Qualität vs. Globalabfrage}

Zu beiden Ansätzen - der Untersuchung spezifischer Einzelmedien oder -gattungen und der Untersuchung eines generalisierten Vertrauens in Journalismus finden sich zwei Varianten der Messung von Vertrauen. Vertrauen in Medien wird einerseits über journalistische Qualitätskriterien operationalisiert, deren wahrgenommene Einhaltung als Indikator für Vertrauen interpretiert wird. Prominent wird dieser Ansatz vertreten in den Arbeiten von Kohring $(2004,2001)$ sowie Kohring und Matthes (2007; 2004) bzw. Matthes und Kohring (2003). Basierend auf der grundlegenden theoretischen Setzung, dass sich das Vertrauen von Rezipient_innen auf die Selektionsleistung des Journalismus richtet, leiten sie ein Messmodell für Vertrauen in Journalismus ab, das vier Dimensionen umfasst (Kohring, 2004, S. 170):

1) Vertrauen in Themenselektivität,

2) Vertrauen in Faktenselektivität,

3) Vertrauen in die Richtigkeit von Beschreibungen und

4) Vertrauen in journalistische Bewertungen.

Das Vertrauen in Themenselektivität richtet sich darauf, dass journalistische Medien diejenigen Themen für die Berichterstattung auswählen, die für das Publikum relevant sind (Vertrauen in Agenda-Setting). Das Vertrauen in Faktenselektivität richtet sich auf die Auswahl von Fakten, die das Thema in einen bestimmten Kontext stellen (Vertrauen in Framing). Vertrauen in die Richtigkeit von Beschreibungen umfasst das Vertrauen in die korrekte Wiedergabe von überprüfbaren Fakten (Glaubwürdigkeit, Kohring, 2004, S. 174). Vertrauen in explizite Bewertungen umfasst schließlich das Vertrauen, das sich auf journalistische Kommentare, Einordnungen und Handlungsempfehlungen bezieht. Kohring (2004) entwickelte dafür eine Skala mit insgesamt 16 Items zur Messung der vier Faktoren, die das Konstrukt zweiter Ordnung, Vertrauen in Journalismus' abbilden. Die Skala bezieht sich allerdings auf das Vertrauen in die Berichterstattung eines Mediums zu einem Thema. Sie umfasst Items wie „dem Thema [...] wird die nötige Aufmerksamkeit geschenkt“, „die Berichterstattung erfolgt aus mehreren Blickwinkeln", „,ie mitgeteilten Informationen sind wahr" oder „die Meinungen der Journalisten sind gut begründet“ (Kohring \& Matthes, 2004, S. 382). 
Als zweiter zentraler Vertreter dieses Ansatzes kann Yariv Tsfati gelten, der unter dem Begriff „media skepticism“ zu Vertrauen in Journalismus gearbeitet hat. Zur Operationalisierung zieht er eine Reihe von Qualitätswahrnehmungen heran und fragt etwa, ob die Befragten die Berichterstattung der Medien für fair, vollständig, vertrauenswürdig und richtig halten. Auch Tsfati verwendet also zur Operationalisierung von Vertrauen in Journalismus konkrete Evaluationen von Qualitätsdimensionen, die sich zwar teilweise mit denen von Kohring überschneiden (z. B. messen beide die Dimension Richtigkeit), allerdings in der konkreten Auswahl der Items auch gravierende Unterschiede aufweisen.

Eine zweite Variante operationalisiert Vertrauen mit einer einfachen globalen Abfrage. Dabei wird meistens mit einem Item nach dem Ausmaß an Vertrauen gefragt, etwa mit Likert-Skalen von wenig bis viel Vertrauen (z. B. Ziegele et al., 2018). Die Messung mit einem Item ist einfach, schnell zu implementieren und verlangt wenig Aufwand bei den Befragten. Im Idealfall provoziert die direkte Frage eine intuitive Reaktion, die das Vertrauenskonstrukt relativ gut abbildet. Allerdings ist dabei nicht klar, was Rezipient_innen genau unter Vertrauen verstehen (Kohring, 2004). Die Antworten können also ganz unterschiedliche, subjektive Konzepte von Vertrauen messen. Außerdem sind Ein-ItemMessungen generell problematisch, da sie keine Kontrolle über Messfehler erlauben und ein Merkmal mit mehreren Items präziser gemessen werden kann (Moosbrugger \& Kelava, 2012, S. 34). Werden konkrete Einschätzungen der Qualität journalistischer Medien vorgelegt, wird klarer, worauf sich das Vertrauensurteil bezieht.

Die Operationalisierung mittels Qualitätswahrnehmungen weist jedoch ebenfalls Probleme auf. Erstens ergibt sich auch hier die bereits aus der Glaubwürdigkeitsforschung bekannte Problematik, dass die zur Operationalisierung verwendeten Qualitätswahrnehmungen in den meisten Fällen recht willkürlich ausgewählt und nur selten theoretisch begründet sind. So verwendet etwa Tsfati in fünf Studien den Begriff „media skepticism“ synonym mit dem Vertrauensbegriff (Tsfati \& Cappella, 2003; Tsfati, 2003a; Tsfati \& Peri, 2006; Tsfati, 2003b; Tsfati \& Cappella, 2005). Für die Messung werden Skalen mit fünf, sieben, acht oder neun Items herangezogen, den Kern bilden jeweils vier bis fünf Items, die ohne theoretische Herleitung aus der Glaubwürdigkeits-Skala von Gaziano und McGrath (1986) entnommen wurden. Je nach Studie werden weitere Items hinzugefügt oder weggelassen. Kohring (2004) liefert als einziger in der Literatur zu Vertrauen eine umfassende theoretische Basis für die Auswahl der Items und kann so eine stringent hergeleitete Skala vorlegen, die sich jedoch nur auf die Berichterstattung eines Mediums zu einem Thema bezieht. Geht es um generalisiertes Vertrauen in Journalismus, handelt es sich dabei aber um eine Engführung, die die wahrgenommene Erfüllung anderer Funktionen von Journalismus nicht berücksichtigt, etwa die korrekte Auswahl unterschiedlicher The- 
men oder die Kritikfunktion des Journalismus (Fortkord \& Fawzi, 2017). Es ist also auch in diesem Forschungsstrang nicht abschließend geklärt, welche Dimensionen das Vertrauenskonstrukt umfasst und welche Qualitätskriterien für eine Operationalisierung von Vertrauen herangezogen werden sollten. Dieses Kernproblem scheint auch kaum lösbar, schließlich könnten sämtliche Wahrnehmungen der Qualität journalistischer Medien vom Design einer Website bis zur wahrgenommenen Ausgewogenheit mit Vertrauen assoziiert sein. Hinzu kommt, dass Qualität keine Eigenschaft der Angebote oder der Medieninstitutionen selbst ist, sondern eine Zuschreibung von außen (Voigt, 2016, S. 21). Was also als qualitätsvoll oder minderwertig gilt, ist je nach Betrachtungsweise unterschiedlich und letztlich immer Ausdruck historisch und kulturell geprägter Vorstellungen. Aktuell wird in der Journalismuspraxis etwa diskutiert, den gesellschaftlichen ,Impact' journalistischer Berichterstattung stärker zu betrachten und dafür die Folgen der Berichterstattung als Erfolgsindikator heranzuziehen. So werden etwa Rücktritte von Politikern oder Gesetzesänderungen aufgrund aufgedeckter Missstände als zählbare Indikatoren für den Erfolg des Journalismus diskutiert (Peteranderl, 2018). Es ist durchaus plausibel, dass auch Teile des Publikums solche Erfolgsfaktoren in den kommenden Jahren zunehmend als Qualitätsdimension erachten und sie für Vertrauen relevant werden. Müsste man sie also in ein qualitätsbasiertes Messmodell von Vertrauen in Journalismus aufnehmen?

Zweitens: In komplexeren Skalen mit mehreren Subdimensionen gehen alle Indikatoren mit der gleichen Gewichtung in die Messung von Vertrauen ein. Die Forschung zu Qualitätswahrnehmungen und Erwartungen an Journalismus hat jedoch gezeigt, dass Menschen durchaus unterschiedliche Erwartungen an Journalismus haben und über unterschiedliche Vorstellungen verfügen, was ihnen am Journalismus wichtig ist (Donsbach, Rentsch, Schielicke, \& Degen, 2009; Trepte, Reinecke, \& Behr, 2008; Arnold, 2009). So legen etwa die meisten Menschen sehr viel weniger Wert auf meinungsbetonte Stücke als auf faktische Informationen. Bestimmte Vertrauensdimensionen in den bisherigen Messinstrumenten sind daher für manche Rezipient_innen vielleicht gänzlich unwichtig, so dass der Beitrag einzelner Qualitätskriterien zum Vertrauenskonstrukt individuell verschieden ist. Vermutlich ist etwa die wahrgenommene faktische Richtigkeit der Berichterstattung ausschlaggebender für Vertrauen als die Wahrnehmung, ob Journalist_innen ihre Meinungen gut begründen. In den gängigen Messmodellen werden diese Kriterien jedoch gleichbehandelt, da sie ja Indikatoren für das latente Konstrukt Vertrauen sind. Aufgrund dieser Gleichsetzung von Vertrauen und Qualitätswahrnehmungen ist bislang ,nicht umfänglich bekannt, was Medienskeptiker und -zyniker [...] konkret den Medien vorwerfen“ und ,in welchem Zusammenhang ihre Medienkritik mit ihrem individuellen Niveau an Medienvertrauen steht" (Jackob, Schultz et al., 2017, S. 131). 
Drittens: Messtheoretisch kann man eine Operationalisierung von Vertrauen mittels Qualitätskriterien als reflektives Messmodell bezeichnen. Diese ,„[...] unterstellen, dass hohe Korrelationen zwischen den Messvariablen bestehen, deren verursachende Größe die betrachtete latente Variable darstellt.“ (Weiber \& Mühlhaus, 2014, S. 42). Vertrauen ist damit das latente Konstrukt, das für die gemeinsame Varianz dieser Indikatoren verantwortlich ist. In solchen Modellen sind die Indikatorvariablen also konzeptuell die Folge der latenten Variable (in diesem Fall von Vertrauen). Diese Konzeption unterstellt also, dass eine Person die Medien als fair, vollständig, richtig etc. wahrnimmt, weil sie ihnen vertraut. Wirth (1999, S. 54) hat auf diese Problematik bereits vor fast 20 Jahren im Kontext der Glaubwürdigkeitsforschung hingewiesen und kommt zu dem Schluss:

Gemäß dieser Vorstellung müßte für jeden Indikator I ein Satz formuliert werden können wie: ,Ein Medium M wird als glaubwürdig wahrgenommen und deswegen gilt $\mathrm{I}^{ }$. Bezogen auf die meisten Glaubwürdigkeitsitems mutet ein derartiger Satz jedoch unplausibel an: Kein Text und kein Moderator wirkt dynamisch, freundlich, aufrichtig, objektiv, informiert oder attraktiv, weil er als glaubwürdig wahrgenommen wird. In der Regel verhält es sich genau anders herum: Aufrichtigkeit und Sachverstand sind gute Gründe, warum einer Botschaft bzw. ihrem Überbringer geglaubt wird.

Viertens: Fragt man nur danach, wie Menschen die Berichterstattung wahrnehmen, beschränkt sich dieses Urteil auf vergangene Erfahrungen mit der Berichterstattung. Vertrauen ist aber auf die Zukunft gerichtet und bedeutet (wie in Kap. 3.3 dargelegt) die Bereitschaft, ein Risiko gegenüber Medien einzugehen. Vertrauen in Medien wird also definiert als die Bereitschaft, Risiken gegenüber Medien einzugehen, aber operationalisiert als Qualitätsbewertung von Medien. Vertrauen ist aber etwas Anderes als Qualitätseinschätzungen von Medien und geht deutlich über die Evaluation eines Bezugsobjekts hinaus. Diese zentrale theoretische Annahme wird in den gängigen Messmodellen jedoch kaum reflektiert. So ist es etwa durchaus denkbar, dass eine Person Qualitätsmängel in journalistischen Medien erkennt, diesen Medien aber dennoch vertraut, weil sie im Sinne der Definition bereit ist, Risiken mit Bezug auf Medien einzugehen (Prochazka \& Schweiger, 2018, S. 15).

Die Messung über Qualitätswahrnehmungen hat außerdem konkrete Konsequenzen für die Forschung zu Vertrauen. So erweist sich Vertrauen in Medien in Experimentalstudien immer wieder als kurzfristig veränderbar, wenn es mit Qualitätswahrnehmungen operationalisiert wird (etwa bei Pingree, Quenette, Tchernev, \& Dickinson, 2013; Pingree et al., 2018; van Duyn \& Collier, 2019; Meier \& Reimer, 2011). Theoretisch ist Vertrauen aber als langfristige, stabile Einstellung konzipiert, die sich durch einen einmaligen experimentellen Stimulus kaum verändern sollte (Tsfati \& Cappella, 2003, S. 507).

Aus diesen Kritikpunkten lassen sich zwei Konsequenzen ziehen. Erstens sind das Konstrukt Vertrauen und das Konstrukt Qualitätswahrnehmung theore- 
tisch und empirisch zu trennen. Zweitens sollten Qualitätswahrnehmungen als Einflussfaktoren auf Vertrauen konzipiert werden, um so den Einfluss möglichst vieler unterschiedlicher Qualitätswahrnehmungen auf Vertrauen in Journalismus in der multivariaten Kontrolle zu untersuchen. So können die relevanten Qualitätseinschätzungen ermittelt und mit anderen Einflussfaktoren verglichen werden.

\subsection{Stand und Entwicklung des Vertrauens in Journalismus in Deutschland}

Wie steht es nun um das Vertrauen in Journalismus in Deutschland? Studien zeigen übereinstimmend solide Vertrauenswerte für journalistische Medien. Im Reuters Digital News Report geben 50 Prozent der Deutschen an, den Nachrichten allgemein meistens zu vertrauen, womit Deutschland im internationalen Vergleich im oberen Drittel angesiedelt ist (Hölig \& Hasebrink, 2017, S. 71). In einem Vergleich der verfügbaren Langzeitdaten zeigen Reinemann et al. (2017, S. 80) außerdem, dass dieses Vertrauen seit den 1990er Jahren relativ stabil geblieben ist: zwischen 30 und 50 Prozent der Befragten geben jeweils an, den Medien eher oder voll und ganz zu vertrauen. Jackob et al. (2019, S. 214) konstatieren für ihre Langzeit-Trendstudie mit den fünf Erhebungszeitpunkten 2008, 2015, 2016, 2017 und 2018 allerdings eine Polarisierung des Journalismusvertrauens (Abbildung 1): Der Anteil der Personen mit hohem Vertrauen in die Medien stieg von 29 Prozent im Jahr 2008 auf 44 Prozent im Jahr 2018. Gleichzeitig hat sich die Gruppe der Personen mit sehr niedrigem Vertrauen mehr als verdoppelt (von 9 Prozent auf 22 Prozent).

In den vergangenen Jahren gaben also weniger Menschen ein mittleres Vertrauen in den Journalismus an, während die Ränder stärker wurden. Es zeigt sich also gegenwärtig in Deutschland eine deutliche Polarisierung beim Vertrauen in journalistische Medien. Auf der einen Seite steht eine relativ große gesellschaftliche Gruppe mit hohem Vertrauen, der eine kleinere, aber medial und öffentlich sehr präsente Gruppe mit sehr geringem Vertrauen entgegensteht. Es sind vermutlich diese Bürger_innen, die aktuell vor allem den Online-Diskurs zu Verfehlungen der Medien prägen (Prochazka \& Schweiger, 2016) und aus deren Mitte die Debatte um die ,Lügenpresse' Aufwind erhält.

Als Initialzündung für diese Phase intensiver Medienkritik in Deutschland gilt die Berichterstattung zur Ukrainekrise im Jahr 2014 (Krüger, 2016, S. 1). Als 


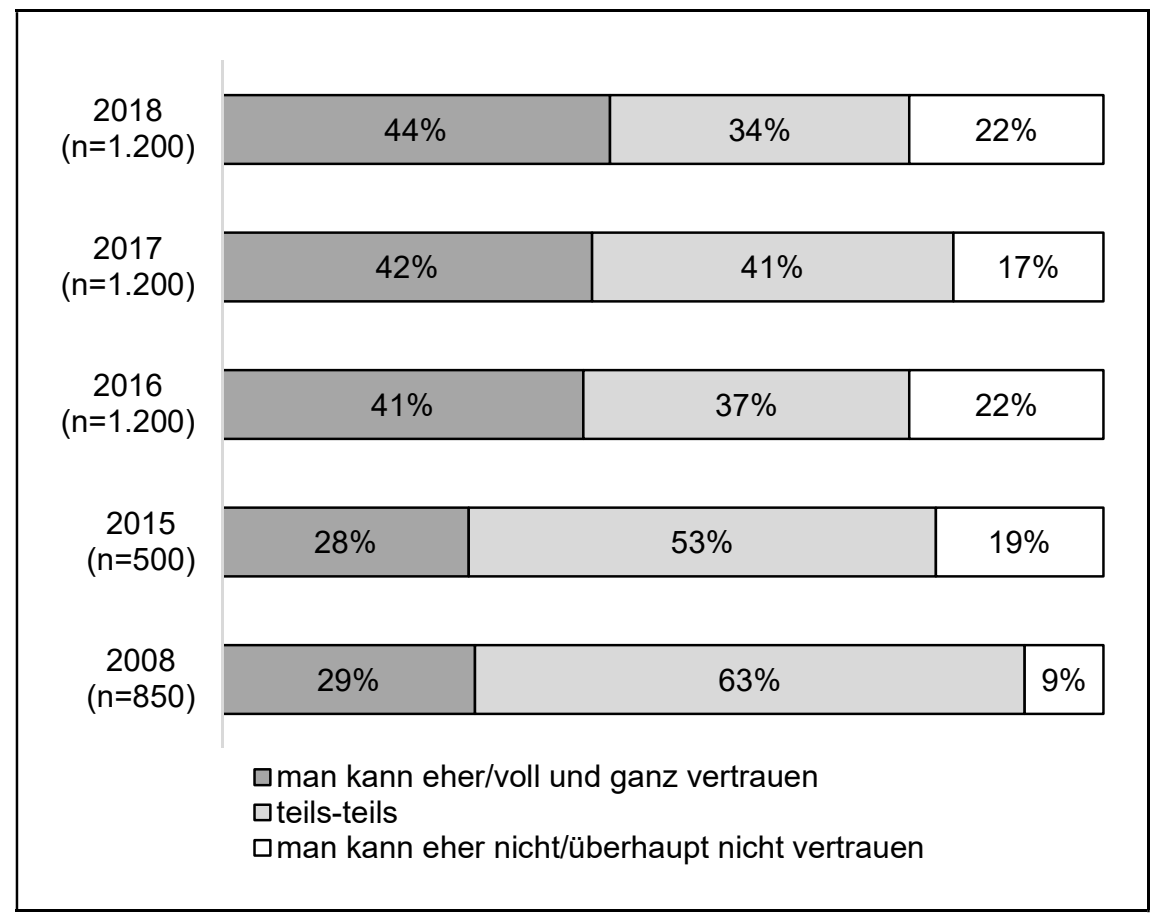

Abbildung 1: Polarisierung des Vertrauens in journalistische Medien.

Frage: „Wenn es um wirklich wichtige Dinge geht - etwa Umweltprobleme, Gesundheitsgefahren, politische Skandale und Krisen - kann man den Medien vertrauen?“،

(Quelle: Jackob et al., 2019, S. 214)

der russisch-ukrainische Konflikt ${ }^{10}$ zu dieser Zeit eskalierte, wurde vor allem in Kommentarspalten auf Nachrichtenseiten und auf sozialen Netzwerkseiten heftige Kritik an der Berichterstattung großer deutscher Leitmedien laut. Ihnen wurde vorgeworfen, über den Krieg in der Ostukraine und die Krimkrise zu einseitig zu berichten, die westliche Perspektive zu stark zu betonen und legitime Positionen Russlands auszublenden (Krüger, 2016, S. 25). In einer Umfrage für das Medienmagazin ZAPP gaben im Dezember 2014 rund 63 Prozent der Befragten an, wenig oder gar kein Vertrauen in die Berichterstattung zur Ukrainekrise zu haben, ein Drittel führte als Grund dafür an, die Berichterstattung sei nicht objektiv bzw. zu einseitig (Infratest Dimap, 2014, S. 9). In der Folgezeit entzündeten sich ähnliche Debatten, vor allem an der Berichterstattung über das Thema Flüchtlin-

10 Zu einer Zusammenfassung des Konflikts siehe LpB (2016), zur Einbettung in breitere (geo)politische Entwicklungen Snyder (2018). 
ge und Einwanderung seit 2015 (Ibis Institut, 2016, S. 8; Arendt, Brosius, \& Hauck, 2017, S. 139). Dort wurden ebenfalls Vorwürfe laut, die Medien würden etwa Flüchtlingskriminalität systematisch verschweigen und die Einwanderung in einem zu positiven Licht darstellen (Haller, 2017, S. 137).

Die Befürchtung eines breiten Vertrauensverlusts journalistischer Medien ist also gemäß den vorliegenden empirischen Daten nicht gerechtfertigt. Im Gegenteil genießt der Journalismus in Deutschland durchaus starken Rückhalt bei einer Mehrheit der Bevölkerung. Vielmehr scheint sich eine kleinere, aber durchaus substanzielle Gruppe zunehmend von journalistischen Medien zu entfremden und in ihren Einstellungen radikaler zu werden. Ihre Argumente erhalten zusätzlich durch tatsächliche Qualitätsmängel der journalistischen Berichterstattung potenziell Auftrieb (siehe Kap. 2.3). Wie in vielen politischen Fragen auch hat sich damit eine Situation entwickelt, in der sich zwei polarisierte Lager gegenüberstehen (Schweiger et al., 2019, S. 73). Was charakterisiert nun diese beiden Lager? Welche Faktoren auf der Individualebene stehen mit hohem bzw. niedrigem Vertrauen in Zusammenhang? Und woher kommt der Vertrauensverlust in Teilen der Bevölkerung bzw. was löst den Vertrauenszuwachs in anderen Teilen aus? Mit diesen Fragen beschäftigen sich die folgenden Kapitel.

Open Access Dieses Kapitel wird unter der Creative Commons Namensnennung 4.0 International Lizenz (http://creativecommons.org/licenses/by/4.0/deed.de) veröffentlicht, welche die Nutzung, Vervielfältigung, Bearbeitung, Verbreitung und Wiedergabe in jeglichem Medium und Format erlaubt, sofern Sie den/die ursprünglichen Autor(en) und die Quelle ordnungsgemäß nennen, einen Link zur Creative Commons Lizenz beifügen und angeben, ob Änderungen vorgenommen wurden.

Die in diesem Kapitel enthaltenen Bilder und sonstiges Drittmaterial unterliegen ebenfalls der genannten Creative Commons Lizenz, sofern sich aus der Abbildungslegende nichts anderes ergibt. Sofern das betreffende Material nicht unter der genannten Creative Commons Lizenz steht und die betreffende Handlung nicht nach gesetzlichen Vorschriften erlaubt ist, ist für die oben aufgeführten Weiterverwendungen des Materials die Einwilligung des jeweiligen Rechteinhabers einzuholen.

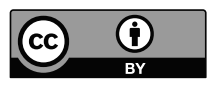

